\title{
High-energy-density electron beam from interaction of two successive laser pulses with subcritical-density plasma
}

\author{
J. W. Wang, ${ }^{1,2, *}$ W. Yu, ${ }^{2}$ M. Y. Yu, ${ }^{3,4, \dagger}$ H. Xu, ${ }^{5}$ J. J. Ju, ${ }^{2}$ S. X. Luan, ${ }^{2}$ \\ M. Murakami, ${ }^{6}$ M. Zepf, ${ }^{1,7}$ and S. Rykovanov ${ }^{1}$ \\ ${ }^{1}$ Helmholtz Institute Jena, Jena 07743, Germany \\ ${ }^{2}$ State Key Laboratory of High Field Laser Physics, Shanghai Institute of Optics and Fine Mechanics, \\ Chinese Academy of Sciences, Shanghai 201800, China \\ ${ }^{3}$ Institute for Fusion Theory and Simulation and the Department of Physics, Zhejiang University, \\ Hangzhou 310027, China \\ ${ }^{4}$ Institute for Theoretical Physics I, Ruhr University, Bochum D-44780, Germany \\ ${ }^{5}$ National Laboratory for Parallel and Distributed Processing, School of Computer Science, \\ National University of Defense Technology, Changsha 410073, China \\ ${ }^{6}$ Institute of Laser Engineering, Osaka University, Osaka 565-0871, Japan \\ ${ }^{7}$ Centre for Plasma Physics, School of Mathematics and Physics, \\ Queen's University Belfast, Belfast BT7 1NN, United Kingdom
}

(Received 11 May 2015; published 4 February 2016)

\begin{abstract}
It is shown by particle-in-cell simulations that a narrow electron beam with high energy and charge density can be generated in a subcritical-density plasma by two consecutive laser pulses. Although the first laser pulse dissipates rapidly, the second pulse can propagate for a long distance in the thin wake channel created by the first pulse and can further accelerate the preaccelerated electrons therein. Given that the second pulse also self-focuses, the resulting electron beam has a narrow waist and high charge and energy densities. Such beams are useful for enhancing the target-back space-charge field in target normal sheath acceleration of ions and bremsstrahlung sources, among others.
\end{abstract}

DOI: 10.1103/PhysRevAccelBeams.19.021301

\section{INTRODUCTION}

As a sufficiently intense laser pulse propagates in a subcritical-density plasma (SCDP), it ponderomotively self-focuses as well as expels a large number of the electrons in its path to form a small cavity with much reduced electron density, followed by a long, somewhat wider, wake channel bounded by a high-density layer of expelled electrons. Inside the tiny cavity leading the wake channel, the electrons are of low density and the laser light therein can directly accelerate them to multi-MeV energies [1-5]. In such an intense laser-plasma interaction (LPI), the light energy is efficiently converted into directed and thermal electron energy, and the laser dissipates rapidly. The electrons in the wake channel behind the tiny cavity are highly nonuniform (turbulent), and locally the density can be comparable to that of the exterior of the SCDP. However, the average electron density in the long wake channel is low, and the latter can survive for many laser periods even after the laser pulse has significantly dissipated or even

\footnotetext{
*J.Wang@gsi.de

'myyu@zju.edu.cn
}

Published by the American Physical Society under the terms of the Creative Commons Attribution 3.0 License. Further distribution of this work must maintain attribution to the author(s) and the published article's title, journal citation, and DOI. disappeared. For a sufficiently short laser pulse, the SCDP ions are almost unperturbed in the front cavity and only slightly perturbed in the wake channel by the irregular space-charge fields. In contrast to the well-known resonant laser-wakefield or bubble acceleration schemes involving extremely low plasma densities, here the accelerated electrons are of relatively low energy and do not show a quasimonoenergetic spectrum, but they are well bunched and have high charge and energy densities. Although such electron beams are not suitable for realizing compact particle accelerators [6], they are useful for enhancing the space-charge field behind a solid-density foil in target normal sheath acceleration (TNSA) of ions [5,7-11], bremsstrahlung sources [12-15], etc.

In this paper, we propose a scheme to produce a highenergy-density (HED) electron beam by enhancing the trapping and acceleration of the SCDP electrons with a second laser pulse that propagates in the thin wake channel of the first laser pulse. The scheme takes advantage of the preaccelerated electrons in the wake channel, which also acts like a tailor-fitted channel or waveguide [16]. As a result, the second laser pulse can propagate for a very long distance in the wake channel and further accelerate the preaccelerated electrons with the self-generated quasistatic electric and magnetic fields. Moreover, because it also self-focuses, the second laser pulse creates an even smaller cavity as well as a narrower wake channel. Eventually, it overtakes the tip of 


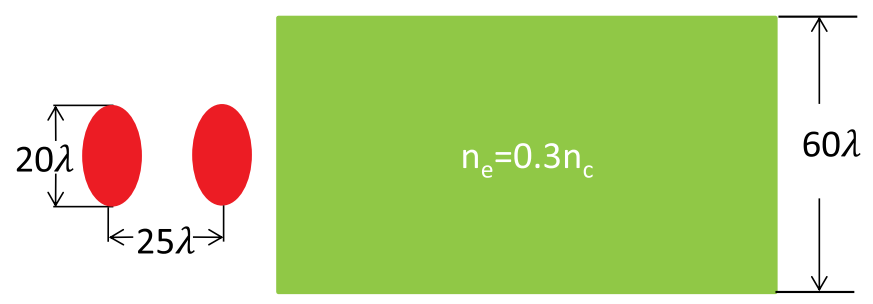

FIG. 1. Proposed scheme. The distance between the two laser pulses is $25 \lambda$.

the first wake channel and enters the pristine SCDP, so that more SCDP electrons can become trapped and accelerated. As a result, the electrons in the wake of the second pulse can reach very high charge and energy densities.

The proposed scheme is illustrated in Fig. 1. Two identical circularly polarized Gaussian laser pulses, each having strength parameter $a_{L}=0.85 \times 10^{-9} \sqrt{I_{L}\left(\mathrm{~W} / \mathrm{cm}^{2}\right)} \lambda(\mu \mathrm{m})=$ 10 , spot radius $b=10 \lambda$, and duration (full width at half maximum) $\tau=5 T_{0}$, propagate from the left into a SCDP with density $n_{e}=0.3 n_{c}$, where $I_{L}$ is the laser intensity, $\lambda=0.8 \mu \mathrm{m}$ and $T_{0}=2.67 \mathrm{fs}$ are the wavelength and period of the laser light, $n_{c}=1.1 \times 10^{21} \lambda(\mu \mathrm{m}) / \mathrm{cm}^{3}$ is the critical density. In reality a capillary can be employed to confine the plasma and prevent its expansion [17-21]. The twodimensional (2D) simulation box is $80 \lambda \times 80 \lambda$. To follow the long-distance propagation of the second laser pulse, a moving window consisting of a $1600 \times 1600$ grid with 36 ions and 36 electrons per cell is used. The ion-electron mass ratio is 1836 .

\section{LONG CAPILLARY AND SCDP}

To investigate the propagation and dissipation characteristics of the two laser pulses in a SCDP, we first consider a long SCDP. The left column in Fig. 2 shows the laser (a) electric field, (b) electron density, and (c) electron energy density at $t=35 T_{0}$, a time when the first laser pulse is propagating steadily in the SCDP and the second laser pulse is just entering it. A part of the local light energy loss in the LPI is compensated by energy concentration due to the laser self-focusing. The ponderomotive force of the first laser pulse compresses as well as pushes aside a large number of the electrons in its path, forming a comoving positively charged quasicavity, which is followed by a wake, as can be seen in Fig. 2(b). We note that instead of an
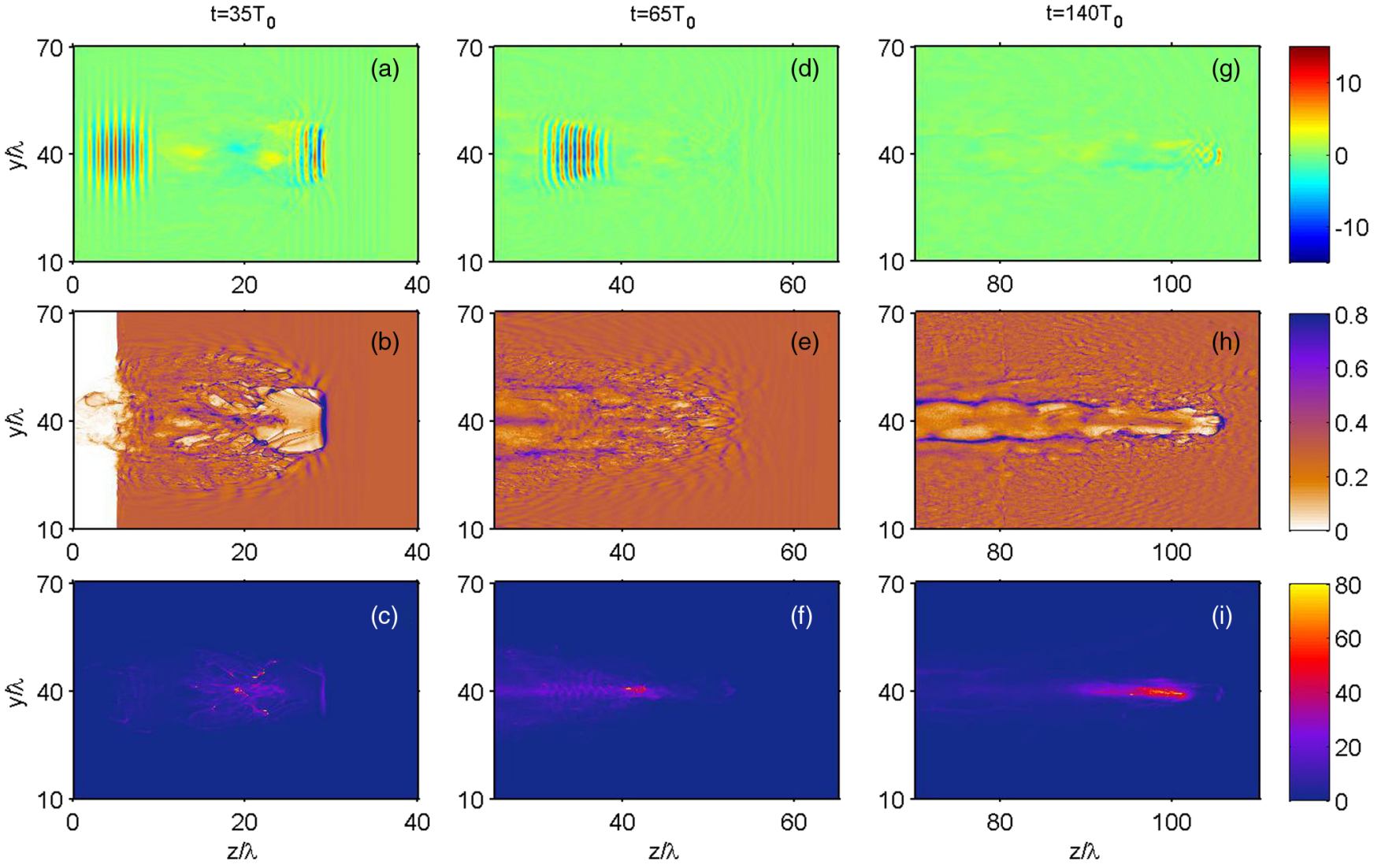

FIG. 2. Evolution of the laser-plasma system at $t=35 T_{0}, 65 T_{0}$, and $140 T_{0}$ (left to right columns). The laser propagates in the $z$ direction. (a), (d), and (g) Laser electric field normalized by $m_{e} \omega c / e$, where $\omega$ is the laser frequency, and $m_{e}$ and $e$ are the electron mass and charge. (b), (e), and (h) Electron density normalized by $n_{c}$. (c), (f), and (i) Electron energy density normalized by $n_{c} m_{e} c^{2}$. The laser strength parameter $a_{L}$ is 10 , the spot radius $b$ is $10 \lambda$, and the duration $\tau$ is $5 T_{0}$. The distance between the two laser pulses is $25 \lambda$. The plasma density is $n_{e}=0.3 n_{c}$. 
almost electron-free wake cavity such as that in the wellknown resonant laser-wakefield acceleration (LWFA) in much lower density plasmas, only a small region with reduced electron density is formed immediately behind the laser pulse and it is in turn followed by a turbulent and open-ended wake channel. The ions in the latter are also (but only slightly; not shown) set into motion in response to the irregular space-charge field. In Fig. 2(c) for the electron energy density, one can see that a HED electron bunch is forming in the axis region $(y \sim 40 \lambda)$ at the back of the quasicavity just before the wake channel. The highly compressed electrons in front of the quasicavity and in the boundary of the wake channel are of high density but low energy. Because of the strong LPI that converts light energy into electron kinetic energy, the propagation range of the first laser pulse in the $\mathrm{SCDP}$ is very limited. We can see in Fig. 2(a) that at $t=35 T_{0}$ it has already weakened significantly.

In the simulation, the distance between the two laser pulses is chosen such that the second laser pulse propagates in a well-formed wake channel created by the first pulse. The thin wake channel acts like a tailor-fitted capillary in that its lateral boundaries are of high electron density formed by the pileup of expelled electrons, and the average
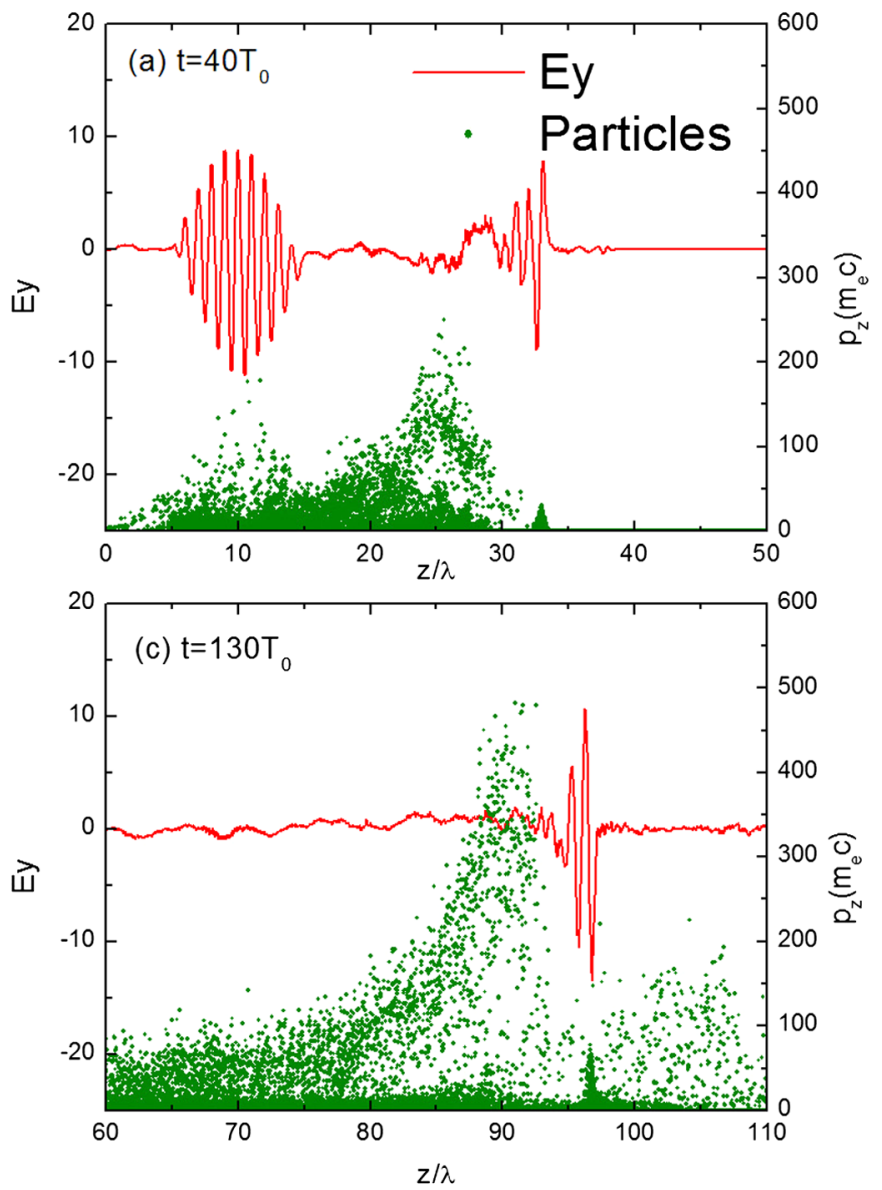

electron density inside decreases toward the channel axis. The oncoming second laser pulse is thus subject to external focusing and self-focusing. The middle column of Fig. 2 shows the interaction scenario at $t=65 T_{0}$. Panels (d) and (e) show that the first laser pulse, now at about $z=55 \lambda$, has become so weak that it is almost invisible. Moreover, because of the much weakened LPI, its speed increases and eventually it propagates forward in the SCDP, leaving the wake channel behind. However, some of the electrons in the wake channel still accelerate and are heated by the space-charge fields or move forward inertially. Meanwhile, quite a few electrons in the channel are directly accelerated by the second laser pulse. Figures 2(d) and 2(f) show that the latter is approaching the HED electron bunch on the wake axis. Note also that, even though the second laser pulse has propagated for over $35 \lambda$ inside the channel, its strength has hardly changed because of the guiding and focusing effects in the wake channel of the first pulse, and the reduced average electron density therein.

As it propagates and focuses in the low average electron density plasma of the first wake channel, the tightly focused second laser pulse creates its own quasicavity and wake channel. A large number of accelerated electrons in the
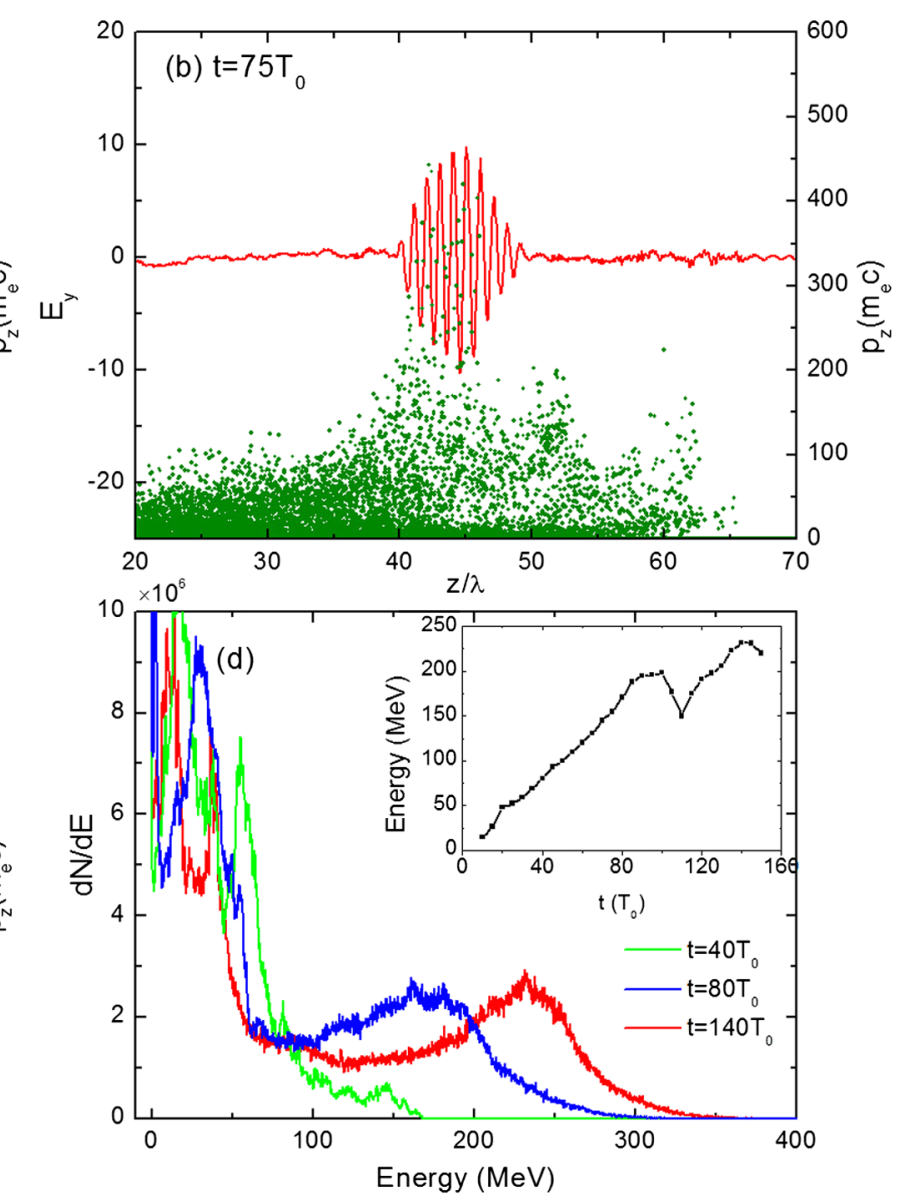

FIG. 3. The transverse electric field normalized by $m_{e} \omega c / e$ along the propagation axis and the electron phase space $\left(z, p_{z}\right)$ at (a) $t=40 T_{0}$, (b) $75 T_{0}$, and (c) $130 T_{0}$. (d) The spectra of the electrons in the channel at different times. The inset shows the time evolution of the energy of the electron bunch. The laser and plasma parameters are the same as those of Fig. 2. 
latter can be trapped in the wakefield of the second laser pulse for a long time and are thus further accelerated. Eventually a thin HED electron beam is formed along the axis. The rightmost column of Fig. 2 displays the laserplasma system at $t=140 T_{0}$, When, after propagating for over $100 \lambda$, the second laser pulse has finally much dissipated. We can see in panel (h) that the new wake channel is only half $(b \sim 5 \lambda)$ the first one $(b \sim 10 \lambda)$. Panel (i) shows that a narrow HED electron beam is propagating along the axis. The narrow wake channel is bounded by high-density layers of ponderomotively expelled electrons both in the front and along the sides of the wake. However, comparison of Figs. 2(h) and 2(i) shows that although the electrons on the wake boundaries are of high number density, they are of very low energy density given that they are rather slow.

The electron acceleration process here consists of two stages. In the first $\left(t<100 T_{0}\right)$, when the second pulse propagates in the wake channel generated by the first pulse, the dominant acceleration mechanism is betatron resonance acceleration, which is essentially direct laser acceleration [22-27]. It differs from ponderomotive-force acceleration $[25,27]$ in that self-generated quasistatic electric and magnetic fields are involved. With the assistance of additional fields, electrons can be accelerated far beyond that by the ponderomotive force of the incident pulse [22-24]. Here the electrons preaccelerated by the space-charge field induced by the first laser pulse are trapped in the ponderomotive bucket of the second pulse, as shown in Figs. 3(a) and 3(b). In the presence of the self-generated quasistatic electric field $E_{y}^{s}$ and magnetic field $B_{x}^{s}$ in the channel, these energetic electrons experience transverse betatron oscillation, as can be seen in Fig. 4. When the betatron frequency is close to the laser frequency, an efficient energy exchange due to betatron resonance between the laser field and electron can take place [23]. The maximum energy gain can be estimated by considering an electron moving in a $p$-polarized plane electromagnetic wave with electric field $E_{y}^{l}=E_{0} \cos \omega_{0}\left(t-z / v_{p h}\right)$ and magnetic field $B_{x}^{l}=$ $-E_{y}^{l} / v_{p h}$ propagating with phase velocity $v_{p h}>c$ in the $z$-direction in the presence of static external electric and magnetic fields given by $E_{y}^{s}=\kappa_{E} y$ and $B_{x}^{s}=\kappa_{B} y$, where $\kappa_{E}$ and $\kappa_{B}$ are constants. In the following discussion, we will use the dimensionless variables $v \rightarrow v / c, t \rightarrow \omega t$, $z \rightarrow z \omega / c, E \rightarrow e E / m \omega c$. In the 2D $(y, z)$ planar geometry, the equation for transverse electron motion is [23]

$$
\frac{d^{2} y}{d t^{2}}+\omega_{\beta}^{2} y=\left[\left(\frac{d y}{d t}\right)^{2}-\left(1-\frac{v_{z}}{v_{p h}}\right)\right] \frac{E^{l}}{\gamma}+\left(\frac{d y}{d t}\right)^{2} \frac{\kappa_{E} y}{\gamma},
$$

where $\omega_{\beta}^{2}=\left(\kappa_{E}+v_{z} \kappa_{B}\right) / \gamma$ is the betatron frequency. Accordingly, resonance occurs if

$$
\omega_{\beta}=1-v_{z} / v_{p h},
$$
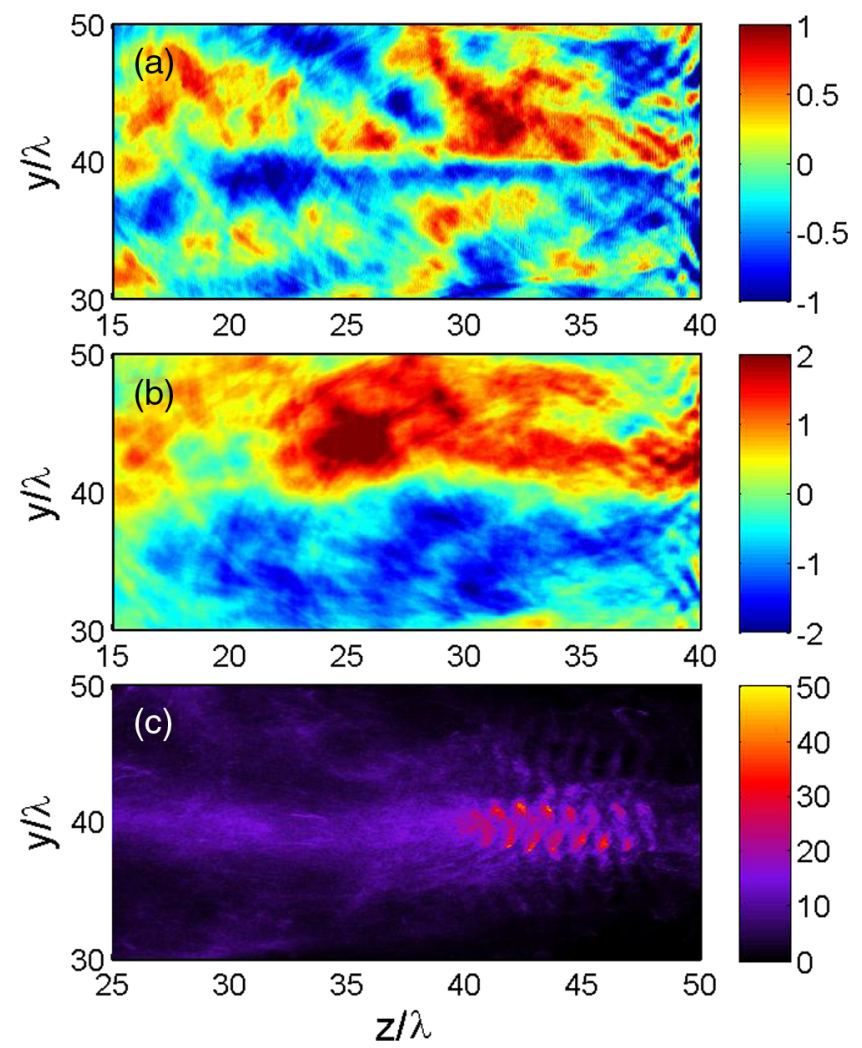

FIG. 4. (a) The self-generated quasistatic electric field $E_{y}^{s}$ normalized by $m_{e} \omega c / e$, (b) the self-generated quasistatic magnetic field $B_{x}^{s}$ normalized by $m_{e} \omega c / e$, and (c) the electron energy density $U$ normalized by $n_{c} m_{e} c^{2}$ in the channel at $t=75 T_{0}$. The laser and plasma parameters are the same as those of Fig. 2.

so that the resonant-electron energy $\gamma$ increases with $\kappa_{E}$ and $\kappa_{B}$ if the quasistatic fields are strong, although not monotonically. For ultrahigh-intensity lasers, a solution of Eq. (2) is

$$
\gamma \sim \frac{\kappa_{E}+\kappa_{B}}{\left(v_{p h}-1\right)^{2}}
$$

which is the maximum energy gain during this acceleration stage. From Eq. (3) we see that the intensity of the static field, induced mainly by the first laser pulse, directly influences the final energy gain by the electron. Moreover, given that $v_{p h}=1 / \sqrt{1-n_{e} / n_{c}}$, the lower channel density indicates that the phase velocity is closer to $c$ and thus a higher energy gain can be achieved. To calculate the maximum energy gain during this stage, one needs the expression of $\kappa_{E}+\kappa_{B}$ and $n_{e}$ in the plasma channel after the first laser. For simplicity we use $\kappa_{E}+\kappa_{B} \simeq 1.6$ and $n_{e} \simeq 0.1$ from the simulation and finally get $\gamma \simeq 550$, which is somewhat larger than the energy gain of about $200 \mathrm{MeV}$ in the simulation in the first stage, as shown in the inset of Fig. 3(d).

In the second acceleration stage $\left(t>100 T_{0}\right)$, the stillintense second pulse enters a pristine plasma and produces its own wakefield. The immediately following 


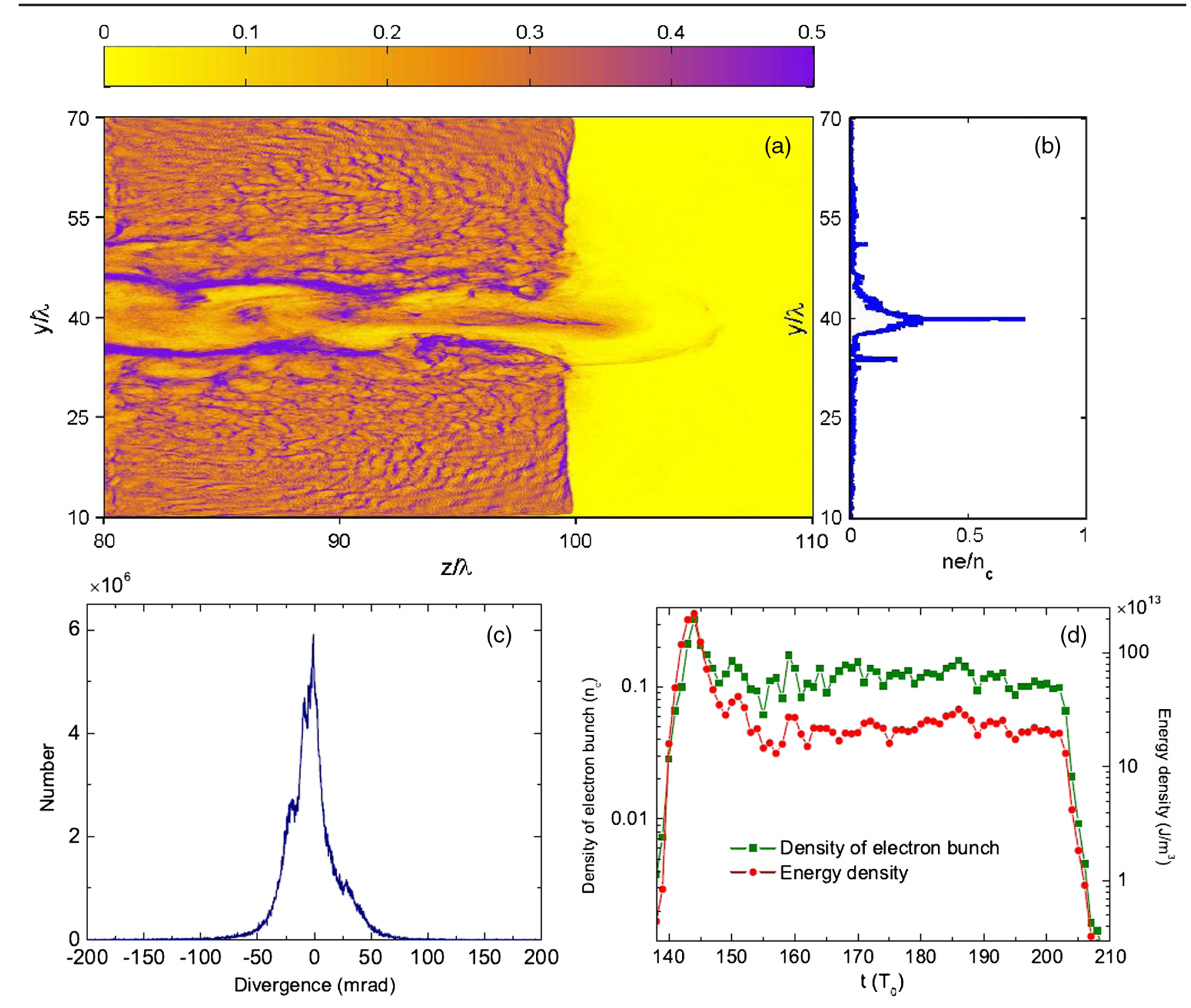

FIG. 5. (a) 2D distribution of the electron density normalized by $n_{c}$. (b) The transverse distribution of the ejected electron beam at $t=140 T_{0}$, when the capillary and the SCDP are terminated at $z=100 \lambda$. (c) Angular spectrum of the electron beam. (d) Evolution of the electron density (green) and electron energy density (red) in the vacuum near the channel opening at $z=102 \lambda$. The oscillations can be attributed to that of the electron bunch, which already existed while still in the channel. The laser and plasma parameters are the same as those of Fig. 2.

preaccelerated electrons enter the latter and are further accelerated, together with the newly trapped backgroundplasma electrons, as can be seen in Fig. 3(c). As a result, both the number and energy of the accelerated electrons are boosted, as shown in Fig. 7(b). The energy gain in this stage can be calculated with the typical wakefield acceleration theory [28]. The maximum axial electric field can be expressed as $E_{\text {zmax }}=\left(k_{p} r_{B} / 2\right) E_{0}$ [29], where $k_{p}$ is the plasma wave number, $r_{B}$ is the cavity radius, and $E_{0}(V / m) \simeq 96 \sqrt{n_{e}\left(\mathrm{~cm}^{-3}\right)}$ is the cold nonrelativistic wave breaking field. The acceleration in such a SCDP case is mainly limited by the pump depletion length $L_{p d}=$ $(\sqrt{2} / \pi) a_{L}\left(\lambda_{p}^{3} / \lambda^{2}\right)$ [30], where $\lambda_{p}$ is the plasma wavelength.
From the simulation one gets $k_{p} r_{B}=6$. Therefore, in theory the energy gain in the second stage is $\Delta \varepsilon_{\text {zmax }}=e E_{\text {zmax }} L_{p d} \simeq 150 \mathrm{MeV}$, which is somewhat larger than the energy gain of about $80 \mathrm{MeV}$ in the simulation. We note that from $t=100 T_{0}$ to $110 T_{0}$ the energy of the newly trapped and accelerated electron bunch decreases. This is because the latter is closing in on the preaccelerated bunch ahead and enters a repulsive field. From the evolution of the electron energy in the inset of Fig. 3(d), one can see that most of the gain is by direct laser acceleration in the first acceleration stage, and the spectral peak of the final (after boosting in the second stage) electron bunch can reach $230 \mathrm{MeV}$. 


\section{BEAM EXTRACTION}

In practice, it is necessary to extract the HED beam from the system. Accordingly, we terminate the SCDP at $z=100 \lambda$, where the energy of the second pulse is almost fully exhausted. Figure 5(a) is for the electron density at $t=140 T_{0}$. We see that the energetic electrons on the axis exit into the vacuum on the rear side as a well-collimated narrow HED jet. Figures 5(b) and 5(c) show that the transverse size of the ejected electron bunch is of the order of microns, with a divergence of $13 \mathrm{mrad}$. The peak density of the tight bunch is $\sim 0.7 n_{c}$. Such tight lateral squeezing can be attributed to the second wake, which is much narrower than the first given that the second laser pulse propagates in a practically tailor-fitted narrow channel produced by the first laser pulse. The collimation of the electron bunch will become worse if the intensity of the laser pulse decreases given that the wake channel will degrade. The charge of the bunch can be estimated to be $Q=e \bar{n}_{e} \pi(d / 2)^{2} l \sim 3 \mathrm{nC}$, where $\bar{n}_{e}=0.286 n_{c}$ is the average electron density in the bunch, $d=3 \lambda$ is the average beam size, $l=12.5 \lambda$ is the beam length, and we have defined the lateral boundaries of the electron bunch as the location where the local density is a half of that at the axis. Thus, the electron yield is almost 1 order of magnitude larger than that from typical wakefield acceleration in very low density plasmas.

The evolution of the electron number and energy densities at the channel opening $(y, z)=(40,102) \lambda$ are given in Fig. 5(d). The density of the out-streaming electrons has a peak value of $0.3 n_{c}$ at $t=143 T_{0}$. Then it decreases to $0.1 n_{c}$ and remains at that level for a relatively long time $150 T_{0}<t<200 T_{0}$, which is over 10 times longer than the laser duration of $\tau=5 T_{0}$, before expansion in the vacuum becomes significant. The electron energy density evolves similarly. Its peak value is $>2 \times 10^{15} \mathrm{~J} / \mathrm{m}^{3}$, also occurring at $t=143 T_{0}$. It then remains at a weaker $\left(2.5 \times 10^{14} \mathrm{~J} / \mathrm{m}^{3}\right)$ level during $150 T_{0}<t<200 T_{0}$. In contrast, recent research indicates that the peak energy density from wakefield acceleration is near $10^{14} \mathrm{~J} / \mathrm{m}^{3}$ [31]. The behavior of the number and energy densities of the HED electron beam at the exit can be expected because the leading electrons in the beam are of the highest velocity and density, followed by the main body of the beam at relatively constant number and energy densities until the end of the beam is reached.

The dependence of the energy and charge of the electron beam on the laser intensity and plasma density is shown in Fig. 6. One can see that the energy increases with the laser intensity but decreases with the plasma density, which is consistent with Eq. (3). The charge of the generated electron bunch is mainly determined by the backgroundplasma density and it weakly increases with the laser intensity. Although the energy gain here is not as high as that in the typical wakefield acceleration, the beam charge (at the nano-Coulomb level) is much higher than
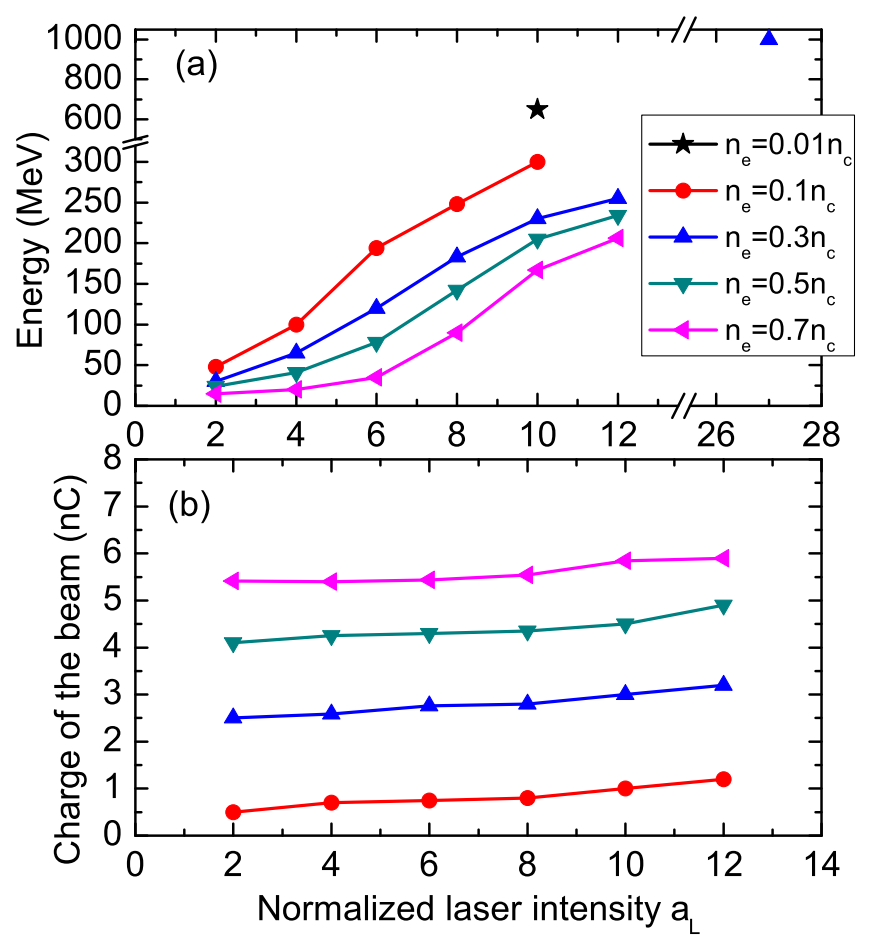

FIG. 6. Dependence of (a) energy and (b) charge of the electron beam on the laser intensity and plasma density. In all cases, the laser spot radius $b=10 \lambda$, duration $\tau=5 T_{0}$, and the distance between the two laser pulses is $25 \lambda$.

that from the latter. It should be noted that the present scheme works in a regime where the plasma density is subcritical and the laser intensity is not sufficiently high to trigger a plasma wakefield in the blow-out regime. For example, electrons can be accelerated to $650 \mathrm{MeV}$ for $a_{L}=10$ and $n_{e}=0.01 n_{c}$ [the black star in Fig. 6(a)], and even to the $\mathrm{GeV}$ level for $a_{L}=28$ and $n_{e}=0.3 n_{c}$ [the blue triangle in Fig. 6(a)]. In both cases the dominant acceleration mechanism is wakefield acceleration.

\section{COMPARISON WITH SINGLE-PULSE ACCELERATION}

In order to see the advantage of the second laser pulse, we compare the results here with that of a single laser pulse having double the intensity but the same duration and total energy. As shown in Fig. 7(a), instead of two $a_{L}=10$ pulses, a single $a_{L}=10 \sqrt{2}$ laser pulse is applied. The simulation is carried out with the same initial plasma parameters. Figure 7(b) shows the evolution of the high-energy (with kinetic energy $>100 \mathrm{MeV}$ ) electrons. The red solid curve is for electron acceleration by the two $a_{L}=10$ laser pulses and the green dashed curve by the single $a_{L}=10 \sqrt{2}$ pulse.

One can see that the two-pulse scheme generates many more high-energy electrons. It should however be pointed out that even without the wake-channel effects, the twopulse scheme should have an advantage in that here the 

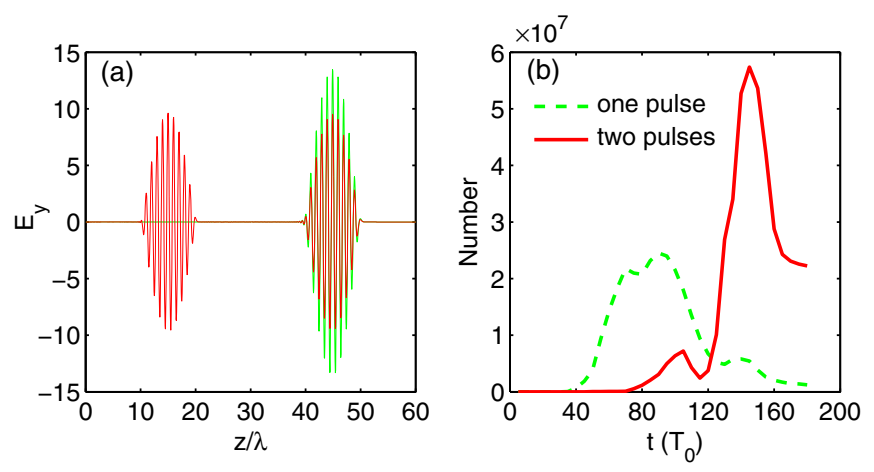

FIG. 7. (a) Profiles of the single and two pulse lasers. The intensity of the single laser pulse is double that of the two pulses, while the duration is the same. (b) Evolution of the number of the high-energy (with kinetic energy $>100 \mathrm{MeV}$ ) electrons for the two cases. The number here is converted from the macroparticle number obtained from the $2 \mathrm{D}$ simulation. The laser duration $\tau=5 T_{0}$, spot radius $b=10 \lambda$, and the plasma density $n_{e}=0.3 n_{c}$. The laser strength parameter is $a_{L}=10$ for the two pulses case, while $a_{L}=10 \sqrt{2}$ for the one pulse case.

driver laser fields have steeper gradients. Thus the total ponderomotive force (scaling with the gradient of the laser intensity) of the two pulses acting on the plasma is higher. In addition, direct acceleration of preaccelerated electrons is much more efficient given that the already-fast electrons remain in the favorable phase of the laser fields for a much longer time $[4,32,33]$.

\section{DISCUSSION}

We have shown that the interaction of two consecutive laser pulses with a SCDP can produce a narrow HED electron beam by enhancing electron trapping and acceleration. The scheme relies on the tapered channel and the preaccelerated electrons created by the short-lived first pulse, such that the second laser pulse can propagate for a long distance in a tailor-fitted channel of low effective density and trap and further accelerate the preaccelerated electrons there by betatron-resonance acceleration. Given that the second laser pulse is channeled, as well as selffocused, its wake is so thin $(<10 \lambda$ across $)$ that the resulting HED electrons are tightly bunched.

It should be emphasized that the present scheme differs considerably from that of the widely investigated laser wakefield acceleration (LWFA) and plasma wakefield acceleration (PWFA) schemes for acceleration of electrons in very low density plasmas to near the light speed [6,34-45]. LWFA and PWFA rely on resonant excitation of intense plasma oscillations when the laser or electronbeam pulse dimension is about half the plasma wavelength. For short pulses the plasma density must thus be very low (e.g., $n \lesssim 10^{-3} n_{c}$ for femtosecond laser pulses) and the laser or electron beam must also be nearly unaffected by the LPI. A small number of electrons can be accelerated to the $\mathrm{GeV}$ level [6,36-40]) by the space-charge electrostatic field in the wake bubble [42]. However, because of the necessarily very low background plasma density and the high electrostatic potential of the wake boundary, even with a second laser pulse, or other novel electron injection schemes, the charge and energy densities of the affected electrons remain rather low [28,46-51]. The present scheme also differs from that invoking near-critical (with $n \sim 1 n_{c}$, both above and below) or even higher density plasmas, where the physics involved is quite different, and the main aim is for ion, instead of electron, acceleration. The accelerated electrons in these schemes are not well bunched and are of relatively low energy density [26,52-55]. Finally, it may be of interest to note that recently a two electron-beam scheme has successfully produced high energy and charge, but also nonmonoenergetic, electron bunches by plasma wakefield acceleration (PWFA) in a very low density plasma [45]. The greatly enhanced PWFA also relies on electron preaccelerated by the first beam.

\section{ACKNOWLEDGMENTS}

We thank Z. Jin and Z. Zhang at the Institute of Laser Engineering, Osaka University, for useful discussions. This work was supported by the National Natural Science Foundation of China (11174303, 11204329, 11304331, 11374262, 11404354), the National Basic Research Program of China (2013CBA01504), the Open Fund of the State Key Laboratory of High Field Laser Physics at the Shanghai Institute of Optics and Fine Mechanics, and the Helmholtz Association (Young Investigator's Group No. VH-NG-1037).

[1] A. Pukhov and J. Meyer-ter-Vehn, Relativistic Magnetic Self-Channeling of Light in Near-Critical Plasma: ThreeDimensional Particle-in-Cell Simulation, Phys. Rev. Lett. 76, 3975 (1996).

[2] A. Pukhov and J. Meyer-ter-Vehn, Relativistic laser-plasma interaction by multi-dimensional particle-in-cell simulations, Phys. Plasmas 5, 1880 (1998).

[3] C. Gahn, G. D. Tsakiris, A. Pukhov, J. Meyer-ter-Vehn, G. Pretzler, P. Thirolf, D. Habs, and K. J. Witte, Multi-MeV Electron Beam Generation by Direct Laser Acceleration in High-Density Plasma Channels, Phys. Rev. Lett. 83, 4772 (1999).

[4] W. Yu, M. Y. Yu, J. X. Ma, Z. M. Sheng, J. Zhang, H. Daido, S. B. Liu, Z. Z. Xu, and R. X. Li, Ponderomotive acceleration of electrons at the focus of high intensity lasers, Phys. Rev. E 61, R2220 (2000).

[5] S. X. Luan, W. Yu, M. Y. Yu, H. B. Zhuo, H. Xu, A. Y. Wong, J. W. Wang, B. F. Shen, and Z. Z. Xu, Target normal sheath acceleration of foil ions by laser-trapped hot electrons from a long subcritical-density preplasma, Phys. Plasmas 21, 123110 (2014).

[6] S. M. Hooker, Developments in laser-driven plasma accelerators, Nat. Photonics 7, 775 (2013). 
[7] S. C. Wilks, A. B. Langdon, T. E. Cowan, M. Roth, M. Singh, S. Hatchett, M. H. Key, D. Pennington, A. MacKinnon, and R. A. Snavely, Energetic proton generation in ultraintense laser-solid interactions, Phys. Plasmas 8, 542 (2001).

[8] H. Daido, M. Nishiuchi, and A. S. Pirozhkov, Review of laser-driven ion sources and their applications, Rep. Prog. Phys. 75, 056401 (2012).

[9] A. Macchi, M. Borghesi, and M. Passoni, Ion acceleration by superintense laser-plasma interaction, Rev. Mod. Phys. 85, 751 (2013).

[10] M. Passoni, C. Perego, A. Sgattoni, and D. Batani, Advances in target normal sheath acceleration theory, Phys. Plasmas 20, 060701 (2013).

[11] H. B. Zhuo, Z. L. Chen, Z. M. Sheng, M. Chen, T. Yabuuchi, M. Tampo, M. Y. Yu, C. T. Zhou, K. A. Tanaka, J. Zhang, and R. Kodama, Collimation of Energetic Electrons from a Laser-Target Interaction by a Magnetized Target Back Plasma Preformed by a Long-Pulse Laser, Phys. Rev. Lett. 112, 215003 (2014).

[12] T. E. Cowan, A. W. Hunt, T. W. Phillips, S. C. Wilks, M. D. Perry, C. Brown, W. Fountain, S. Hatchett, J. Johnson, M. H. Key, T. Parnell, D. M. Pennington, R. A. Snavely, and Y. Takahashi, Photonuclear Fission from High Energy Electrons from Ultraintense Laser-Solid Interactions, Phys. Rev. Lett. 84, 903 (2000).

[13] K. W. D. Ledingham et al., Photonuclear Physics when a Multiterawatt Laser Pulse Interacts with Solid Targets, Phys. Rev. Lett. 84, 899 (2000).

[14] Y. Glinec, J. Faure, L. Le Dain, S. Darbon, T. Hosokai, J. J. Santos, E. Lefebvre, J. P. Rousseau, F. Burgy, B. Mercier, and V. Malka, High-Resolution $\gamma$-Ray Radiography Produced by a Laser-Plasma Driven Electron Source, Phys. Rev. Lett. 94, 025003 (2005).

[15] V. Ramanathan, S. Banerjee, N. Powers, N. Cunningham, N. A. Chandler-Smith, K. Zhao, K. Brown, D. Umstadter, S. Clarke, S. Pozzi, J. Beene, C. R. Vane, and D. Schultz, Submillimeter-resolution radiography of shielded structures with laser-accelerated electron beams, Phys. Rev. ST Accel. Beams 13, 104701 (2010).

[16] J. H. Cooley, T. M. Antonsen, H. M. Milchberg, J. Fan, L. Margolin, and L. Pyatnitskii, Parametric instability in the formation of plasma waveguides, Phys. Rev. E 73, 036404 (2006).

[17] A. Butler, D. J. Spence, and S. M. Hooker, Guiding of High-Intensity Laser Pulses with a Hydrogen-Filled Capillary Discharge Waveguide, Phys. Rev. Lett. 89, 185003 (2002).

[18] W. P. Leemans, B. Nagler, A. J. Gonsalves, Cs. Tóth, K. Nakamura, C. G. R. Geddes, E. Esarey, C. B. Schroeder, and S. M. Hooker, GeV electron beams from a centimetrescale accelerator, Nat. Phys. 2, 696 (2006).

[19] C. E. Clayton, J. E. Ralph, F. Albert, R. A. Fonseca, S. H. Glenzer, C. Joshi, W. Lu, K. A. Marsh, S. F. Martins, W. B. Mori, A. Pak, F. S. Tsung, B. B. Pollock, J. S. Ross, L. O. Silva, and D. H. Froula, Self-Guided Laser Wakefield Acceleration beyond $1 \mathrm{GeV}$ Using Ionization-Induced Injection, Phys. Rev. Lett. 105, 105003 (2010).

[20] J. W. Wang, W. Yu, M. Y. Yu, A. L. Lei, X. Wang, V. K. Senecha, X. G. Wang, M. Murakami, and K. Mima, Guiding of intense laser pulse in uniform plasmas and preformed plasma channels, Phys. Plasmas 17, 103109 (2010).

[21] X. M. Wang and et al., Quasi-monoenergetic laser-plasma acceleration of electrons to $2 \mathrm{GeV}$, Nat. Commun. 4, 1988 (2013).

[22] J. Meyer-ter-Vehn and Z. M. Sheng, On electron acceleration by intense laser pulses in the presence of a stochastic field, Phys. Plasmas 6, 641 (1999).

[23] A. Pukhov, Z.-M. Sheng, and J. Meyer-Ter-Vehn, Particle acceleration in relativistic laser channels, Phys. Plasmas 6 , 2847 (1999).

[24] Z.-M. Sheng, K. Mima, J. Zhang, and J. Meyer-ter-Vehn, Efficient acceleration of electrons with counterpropagating intense laser pulses in vacuum and underdense plasma, Phys. Rev. E 69, 016407 (2004).

[25] W.-M. Wang, Z.-M. Sheng, Y.-T. Li, L.-M. Chen, S. Kawata, and J. Zhang, Monoenergetic electron bunches generated from thin solid foils irradiated by ultrashort, ultraintense circularly polarized lasers, Phys. Rev. ST Accel. Beams 13, 071301 (2010).

[26] J. W. Wang, M. Murakami, S. M. Weng, H. Ruhl, S. X. Luan, and W. Yu, Monoenergetic collimated nanoCoulomb electron beams driven by crossed laser beams, Appl. Phys. Lett. 103, 024105 (2013).

[27] C. K. Lau, P. C. Yeh, O. Luk, J. McClenaghan, T. Ebisuzaki, and T. Tajima, Ponderomotive acceleration by relativistic waves, Phys. Rev. ST Accel. Beams 18, 024401 (2015).

[28] E. Esarey, C. B. Schroeder, and W. P. Leemans, Physics of laser-driven plasma-based electron accelerators, Rev. Mod. Phys. 81, 1229 (2009).

[29] W. Lu, C. Juang, M. Zhou, M. Tzoufras, F. S. Tsung, W. B. Mori, and T. Katsouleas, A nonlinear theory for multidimensional relativistic plasma wave wakefields, Phys. Plasmas 13, 056709 (2006).

[30] B. A. Shadwick, C. B. Schroeder, and E. Esarey, Nonlinear laser energy depletion in laser-plasma accelerators, Phys. Plasmas 16, 056704 (2009).

[31] W. P. Leemans, A. J. Gonsalves, H.-S. Mao, K. Nakamura, C. Benedetti, C. B. Schroeder, C. Tth, J. Daniels, D. E. Mittelberger, S. S. Bulanov, J.-L. Vay, C. G. R. Geddes, and E. Esarey, Multi-GeV Electron Beams from CapillaryDischarge-Guided Subpetawatt Laser Pulses in the SelfTrapping Regime, Phys. Rev. Lett. 113, 245002 (2014).

[32] Z.-M. Sheng, L. W. Zhu, and M. Y. Yu, Electron acceleration by an intense laser pulse with echelon phase modulation, New J. Phys. 12, 013011 (2010).

[33] L. W. Zhu, Z.-M. Sheng, and M. Y. Yu, Direct acceleration of electrons by a circular polarized laser pulse with phase modulation, Phys. Plasmas 20, 113112 (2013).

[34] T. Tajima and J. M. Dawson, Laser Electron Accelerator, Phys. Rev. Lett. 43, 267 (1979).

[35] P. Mora and T. M. Antonsen, Electron cavitation and acceleration in the wake of an ultraintense, self-focused laser pulse, Phys. Rev. E 53, R2068 (1996).

[36] J. Faure, Y. Glinec, A. Pukhov, S. Kiselev, S. Gordienko, E. Lefebvre, J.-P. Rousseau, F. Burgy, and V. Malka, A laser-plasma accelerator producing monoenergetic electron beams, Nature (London) 431, 541 (2004).

[37] W. P. Leemans, B. Nagler, A. J. Gonsalves, Cs. Toth, K. Nakamura, C. G. R. Geddes, E. Esarey, C. B. Schroeder, 
and S. M. Hooker, GeV electron beams from a centimetrescale accelerator, Nat. Phys. 2, 696 (2006).

[38] W. Lu, M. Tzoufras, C. Joshi, F. S. Tsung, W. B. Mori, J. Vieira, R. A. Fonseca, and L. O. Silva, Generating multi$\mathrm{GeV}$ electron bunches using single stage laser wakefield acceleration in a 3D nonlinear regime, Phys. Rev. ST Accel. Beams 10, 061301 (2007).

[39] M. Fuchs, R. Weingartner, A. Popp, Z. Major, S. Becker, J. Osterhoff, I. Cortrie, B. Zeitler, R. Horlein, G. D. Tsakiris, U. Schramm, T. P. Rowlands-Rees, S. M. Hooker, D. Habs, F. Krausz, S. Karsch, and F. Gruner, Laser-driven soft-X-ray undulator source, Nat. Phys. 5, 826 (2009).

[40] A. Borot, A. Malvache, X. Chen, A. Jullien, J.-P. Geindre, P. Audebert, G. Mourou, F. Quéré, and R. Lopez-Martens, Attosecond control of collective electron motion in plasmas, Nat. Phys. 8, 416 (2012).

[41] M. S. Kim, D. G. Jang, T. H. Lee, I. H. Nam, I. W. Lee, and H. Suk, Characteristics of a tapered capillary plasma waveguide for laser wakefield acceleration, Appl. Phys. Lett. 102, 204103 (2013).

[42] S. Gordienko and A. Pukhov, Scalings for ultrarelativistic laser plasmas and quasimonoenergetic electrons, Phys. Plasmas 12, 043109 (2005).

[43] C. Ren, B. J. Duda, R. G. Hemker, W. B. Mori, T. Katsouleas, T. M. Antonsen Jr., and P. Mora, Compressing and focusing a short laser pulse by a thin plasma lens, Phys. Rev. E 63, 026411 (2001).

[44] L. Silva and J. T. Mendonça, Kinetic theory of photon acceleration: Time-dependent spectral evolution of ultrashort laser pulses, Phys. Rev. E 57, 3423 (1998).

[45] M. Litos et al., High-efficiency acceleration of an electron beam in a plasma wakefield accelerator, Nature (London) 515, 92 (2014).

[46] L. Plaja and L. Roso, Analytical description of a plasma diffraction grating induced by two crossed laser beams, Phys. Rev. E 56, 7142 (1997).
[47] Q. L. Dong, Z. M. Sheng, and J. Zhang, Self-focusing and merging of two copropagating laser beams in underdense plasma, Phys. Rev. E 66, 027402 (2002).

[48] J. Faure, C. Rechatin, A. Norlin, A. Lifschitz, Y. Glinec, and V. Malka, Controlled injection and acceleration of electrons in plasma wakefields by colliding laser pulses, Nature (London) 444, 737 (2006).

[49] R.H.H. Scott et al., Controlling Fast-Electron-Beam Divergence Using Two Laser Pulses, Phys. Rev. Lett. 109, 015001 (2012).

[50] Z. G. Deng, L. Yang, C. T. Zhou, M. Y. Yu, H. P. Ying, and X. G. Wang, Collision of counterpropagating laser-excited wake bubbles, Phys. Rev. E 89, 063101 (2014).

[51] G. Golovin, S. Chen, N. Powers, C. Liu, S. Banerjee, J. Zhang, M. Zeng, Z. Sheng, and D. Umstadter, Tunable monoenergetic electron beams from independently controllable laser-wakefield acceleration and injection, Phys. Rev. ST Accel. Beams 18, 011301 (2015).

[52] S. C. Wilks, W. L. Kruer, M. Tabak, and A. B. Langdon, Absorption of Ultra-Intense Laser Pulses, Phys. Rev. Lett. 69, 1383 (1992).

[53] M. Borghesi, A. J. MacKinnon, L. Barringer, R. Gaillard, L. A. Gizzi, C. Meyer, O. Willi, A. Pukhov, and J. Meyer-ter-Vehn, Relativistic Channeling of a Picosecond Laser Pulse in a Near-Critical Preformed Plasma, Phys. Rev. Lett. 78, 879 (1997).

[54] J. Fuchs, G. Malka, J. C Adams, F. Amiranoff, S. D. Baton, N. Blanchot, A. Héron, G. Laval, J. L. Miquel, P. Mora, H. Pépin, and C. Rousseaux, Dynamics of Subpicosecond Relativistic Laser Pulse Self-Channeling in an Underdense Preformed Plasma, Phys. Rev. Lett. 80, 1658 (1998).

[55] L. Willingale, P. M. Nilson, A. G. R. Thomas, S. S. Bulanov, A. Maksimchuk, W. Nazarov, T. C. Sangster, C. Stoeckl, and K. Krushelnick, High-power, kilojoule laser interactions with near-critical density plasma, Phys. Plasmas 18, 056706 (2011). 